\title{
A Survey on Coverage Problem in Wireless Sensor Network
}

\author{
Priti K. Hirani \\ M-Tech CS (Student) \\ Jayoti Vidhyapeeth Women's \\ University, Jaipur, India
}

\author{
Manali Singh \\ Assistant Professor \\ Jayoti Vidhapeeth Women's \\ University, Jaipur, India
}

\begin{abstract}
Wireless sensor networks are rapidly growing for research and commercial development which is used for monitor a given field of interest to change the environment wireless sensor network broadly used in health care, national security, surveillance, and military. We survey the current work on the coverage problem in wireless sensor network. There are two types of coverage. One is area coverage and another is target coverage. The issue of quality of service is related to the coverage problem in wireless sensor network. The coverage concepts depend from several points of view due to a variety of sensor node. Coverage in wireless sensor network is usually defined as a measure of how well and for how long the sensor nodes are able to observe the physical. In this paper present coverage problem in Wireless Sensor Network.
\end{abstract}

\section{General Terms}

Coverage Problems, Coverage Strategies.

\section{Keywords}

Wireless sensor network, coverage, network lifetime.

\section{INTRODUCTION}

In recent year wireless sensor network has been increasing interest in this field. The wireless sensor includes many sensors which are applicable in supervision and security environment. They consist of sensor node deployed over a geographical area for monitoring physical phenomena like temperature, humidity, vibration and so on. A wireless sensor network composes of the large scale of sensor device which is called sensor node. The sensor node consists five major components. Sensor, Memory, Radio, Processor and Power source. All other component sensor nodes are composed of the power source. The power source of as sensor node is batter based is not able to recharge or replace batteries of sensor node due to a number of factors like a large number of sensor nodes, distribution of node in remote access, desert or hostile are etc. according to research the radio is most power consuming. Network life time is continuous time duration for which all the target in given area. Coverage and connectivity are two major issues for Quality of Service in the wireless sensor network. QoS of the network can be measured through the energy consumption to execute the service. The most important factor for developing wireless sensor network is to consider the coverage problem and decreasing the energy use of the sensor. Coverage is important for a sensor network maintain connectivity. Connectivity can be defined as the ability of the sensor node to reach data link. Each node has a communication rage which has defined the area which another node can be located in order to receive data. The Sensor in a network can gather information from an interesting region of observation and transmit this collected information to the base station. There are two types of data sent. 1. event-driven, 2. On- demand. In event driven data are sent to the base station when one or more sensor detects an event. In On -demand, data sent from the sensor to the base station on request.

In these paper is organized follows: section: coverage and connectivity issue in wireless sensor network, Section: we present different kind of strategies, Section: conclusion

\section{COVERAGE AND CONNECTIVITY ISSUES}

The area of coverage and connectivity are closely related to each other. They have been combining two into a single algorithm. An important principle to consider that if the communication range of the sensor is at least twice of that of the sensing range then coverage of area is implies connectivity. Optimal resource management and assuring reliable QoS are two of most fundamental requirement in wireless sensor network. The issue of how well each point in the sensing field is covered. However, server of resource constraint and hostile environment condition. It is non trial to design an efficient development strategy that would reduce computation, minimize cost and minimize node to node communication. It is necessary to define a precise measure of efficient coverage that will input overall system performance.

There are different types of coverage.

Blanket coverage: Sensor node that maximizes the detection rate of the target.

Barrier coverage: Sensor node that minimizes the probability of undetected penetration through the barrier.

Sleep coverage: A number of sensor node move across a sensing field, such that it addresses specified balance between the detection rate and minimizing the number of miss detection. 


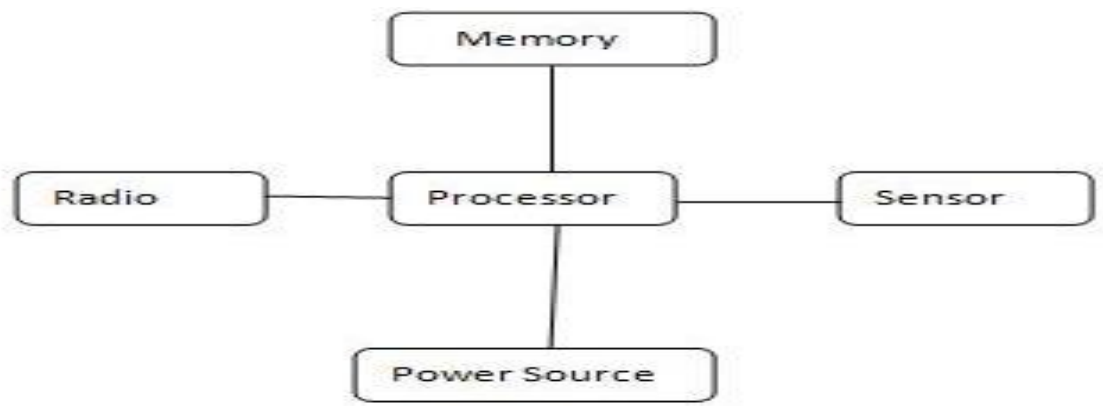

Fig 1: Sensor components

We present the coverage configuration protocol. The protocol attempt to maximize the number of nodes that put into sleep mode. They use a Voronoi diagram to prove that Coverage implies connectivity when $R_{c} \geq 2 R_{s}$.they also proved the convex region, the connectivity is $2_{\mathrm{kj}}$ if $R_{c} \geq 2 R_{s}$, to prove the coverage the author focus on the boundary of the sensor range. The node in CCP (Coverage Configuration Protocol) can be in one of three states. ACTIVE, LISTEN, SLEEP. Each node will send periodically send out Hello packets with its location and static. Status the node will compile a list of each of its neighbor when it is in LISTEN state. It covered sensing area if neighbors then it will transaction in to SLEEP mode. The CCP protocol does not guarantee connectivity when $R_{c} \geq 2 R_{s}$.

Another protocol that attempt to coverage and connectivity is Optional Geographical Density Control (OGDC) in CCP that coverage implies connectivity when $R_{c} \geq 2 R_{s}$.the node in ODGC can be any of three states. ON, OFF, UNDECIDED. The nodes start off a UNDECIDED and then transition to either ON or OFF for steady phase. These two protocols are very similar. The OGDC protocol compares their work with CCP. The find that their protocol extends the nodes lifetimes much better than the CCP. Both of protocols are useful for dense sensor network. If there is not high degree of redundancy then overhead of implement of they would not be worth the benefits.

Some recent were in this field. They approach the problem connected coverage in sensor network with heterogeneous sensing ranges. Helly theorem to help determine coverage area. They defined the sensor states as READY, WAITING and RUNNING. A sensor states that are waiting has its radio turnoff to stay in that state. When a sensor id Ready state is waiting to be woken up by awake a message from a running
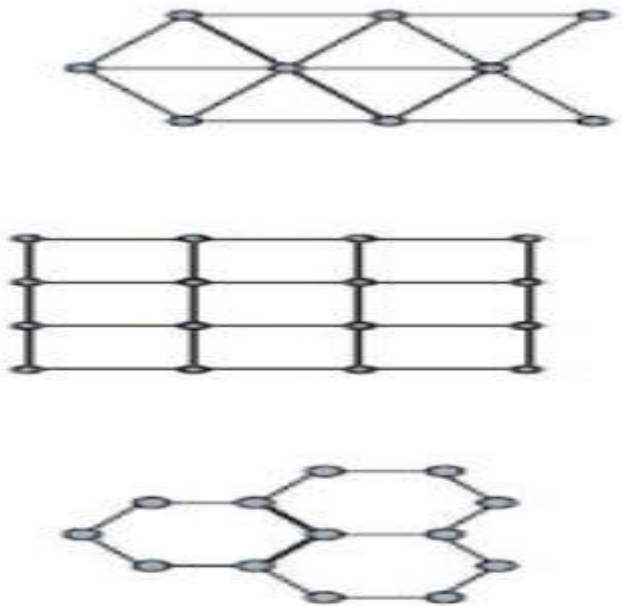

node able to communicate and sense. They implement both centralized, distributed connected K-coverage protocol.

\section{COVERAGE STRATEGIES}

Coverage strategies used for solving coverage problems in wireless sensor network. The strategies are divided into three categories force base, Grid based, Computational geometry based.

\subsection{Force based}

Force base development a strategy rely on the sensor mobility, using virtual repulsive and attractive force the sensor force to move away or awards each other so that full coverage is achieved. Each sensor node exerts an attractive or repulsive forced is exerted. Each neighboring sensor according to the distance between them in order to reach the target distance. A force can be described as a vector and overall force is then the result if vector addition.

Following condition of force function:

Inversion relation: this is implies that the force is inversely proposal to nodes distance.

Upper bound: $f_{\max }$ is maximum force. This is set an upper bound on force.

Lower bound: $f\left(d_{i j}\right)=0$, if $d_{i j}>R_{e}$, this is indicated that only neighbor exert force.

We also introduced two rules for node movement

\subsubsection{Oscillation Check}

If one node move back and forth between similar location many times, that these nodes are regarded to in oscillation states.
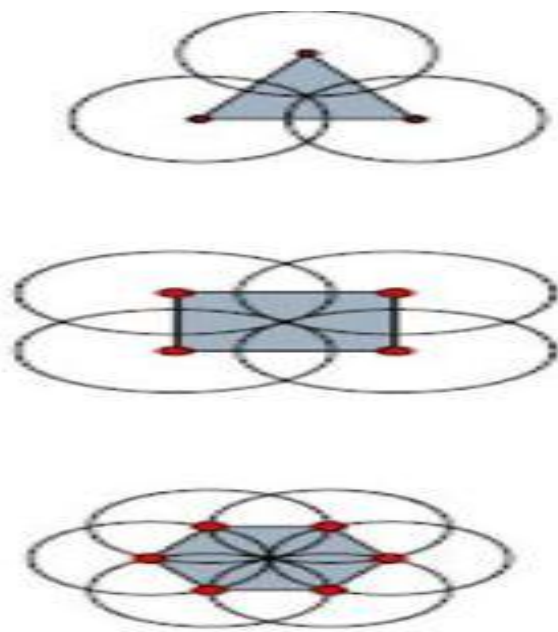

Fig 2: Type of Grids 


\subsubsection{Stability Check}

If the node moving distance of node is less than a predefined threshold for several times, then this node can be considered to have reached a stable state.

\subsection{Grid based}

Grid points are used in two ways in wireless sensor network deployment either to measurement coverage as used in VFA or determine the sensor position. In these approach sensor will redeploy according to a predefined grid. Triangular Lattice, Square or Hexagonal grid. The triangular lattice is preferred because it requires the smallest number of sensor network density. But in sparse network large grid size is better than it will avoid overlapping of sensor sensing range therefore ensuring full utilization of their sensor capabilities. The grid can be used in robotic where robotic moves according to defined rules and deploy on sensor achieve the full coverage of area.

\subsection{Computational Geometry based}

Computational geometry is frequently used in wireless sensor network for coverage optimization the most frequently used computational geometry approach are a Voronoi graph and Delaunay triangulation. In Voronoi diagraph id partition of sites, in such way that point inside the polygon. Voronoi graph can be used as one of the sampling methods in determining wireless sensor network act as the sites.

Voronoi diagram and Delaunay triangulation are used in to estimate the worst and best coverage.

\subsubsection{Worst case coverage}

A path where an introduced can go through with the least probability of being detected, and maximum support part.

\subsubsection{Best case coverage}

A path with the highest coverage. The work proved that a maximum breach path must lie on the edges of Voronoi diagram.

\section{CONCLUSION}

Coverage is a wireless sensor network can be thought of as how well the wireless sensor network is able to monitor a particular field internet. The coverage strategies are authorized into three different approaches: force based, Grid based, Computational geometry based. In these papers we have attempted to overview of the work that has been done to address the coverage problem in wireless sensor network. Also, used a hybrid of the PSO and DE algorithm for area coverage and to increase the lifetime of the network.

\section{REFERENCE}

[1] H.Zhang. "Energy -Balance Heuristic Distributed Algorithm for Target Coverage in Wireless Sensor Network with Adjustable Sensing Range”, 2009 Asia Pacific Conference on Information.

[2] Ghosh, A. and Das, S.K, "Coverage and Connectivity Issues in Wireless Sensor Network" in Shorey, R., Ananda, A.L, Chan, M.C. and Ooi, W.T "Mobile, Wireless, and Sensor Network: Technology, Application and Future Directions”, John Wiley \& sons(2006)

[3] Zou, Y. and Krishnedu Chakrabarty "Sensor deployment and target localization based on virtual forces" Twenty Second Annual Joint Conference of the IEEE Computer and Communication Societies. IEEE Volume 2, 2003.

[4] V. Raghunathan, C. Schurgers, S. Park, and M.B. Srivastava, Energy - Aware wireless Micro Sensor Networks, IEEE Signal Processing Magazine, 2002.

[5] M. T. Thai, Y. Li, F. Wang, and D-Z,Du, Minimum Coverage Breach and Maximum Network Lifetime in Wireless Sensor Network"

[6] S. Yang, F. Dai, M. Cardei, J. Wu, and F. Patterson, "On Connected Multiple Point coverage in Wireless Sensor Networks", Journal of Network and Computer Applications, Volume 32 issue 4(July 2009).

[7] J. Chen and X.Koutsoukos. "Survey on Coverage Problem in Wireless ad hoc Sensor Networks", In IEEE communications Volume .9(Nov. 2005).

[8] Chakrabarty, K., Iyengar, S.S., Qi, H. and Cho, E. "Grid Coverage for Surveillance and Target Location in Distributed Sensor Networks" IEEE Transactions on Computer vol. 51 (2002).

[9] Wu, C.H, Lee, K.C, Yeh-Ching chung, Y.C “A Delaunay triangulation based method for wireless sensor networks deployment" 12 th International Conference On parallel and distributed Systems, volume 1, (July 2006).

[10] Y. Jennifer, M. Biswanath, and G. Dipak, "Wireless Sensor Network Survey", Computer Networks, 2008 